

\title{
Las configuraciones territoriales de movilidad, o el espacio como lenguaje político
}

Odile Hoffmann, Abelardo Morales Gamboa

\section{To cite this version:}

Odile Hoffmann, Abelardo Morales Gamboa. Las configuraciones territoriales de movilidad, o el espacio como lenguaje político. El territorio como recurso: movilidad y apropiación del espacio en México y Centroamérica, 2018. halshs-02304049

\section{HAL Id: halshs-02304049 \\ https://shs.hal.science/halshs-02304049}

Submitted on 2 Oct 2019

HAL is a multi-disciplinary open access archive for the deposit and dissemination of scientific research documents, whether they are published or not. The documents may come from teaching and research institutions in France or abroad, or from public or private research centers.
L'archive ouverte pluridisciplinaire HAL, est destinée au dépôt et à la diffusion de documents scientifiques de niveau recherche, publiés ou non, émanant des établissements d'enseignement et de recherche français ou étrangers, des laboratoires publics ou privés. 


\section{VERSION ANTES DE EDICION}

\section{REFERENCIA}

Hoffmann, Odile (2018). « Las configuraciones territoriales de movilidad, o el espacio como lenguaje político », pp23-40 en O.Hoffmann et A.Morales (coord.), El territorio como recurso: movilidad y apropiación del espacio en México y Centroamérica, San José Costa Rica : UNA-FLASO-IRD, 240p.

Las configuraciones territoriales de movilidad, o el espacio como lenguaje político

Odile Hoffmann

2017

\section{Introducción}

Partiendo del postulado de que las relaciones tejidas con y alrededor del espacio se recomponen cuando se modifican los entornos y ambientes de vida, buscamos entender cómo la experiencia de movilidad (migración, circulación, retornos) redibuja las categorías de experiencia, en particular las categorías de prácticas y representaciones espaciales de los sujetos-habitantes-migrantes. Entendemos que el espacio no es mero soporte ni contexto, que sólo existe en tanto que concebido, comprendido y producido por actores sociales y sociedades y, de manera compleja y diferencial, dentro de estas sociedades (lo desarrollo más adelante). Pero, además de ser una producción y una construcción social, el espacio también es portador de sentido y, por lo tanto, productor de comportamientos o acciones. Es decir, el espacio es a la vez producto y productor de sentido social, se sitúa en el interfaz de la innovación y de la reproducción social y política. Comprender mejor las relaciones que se tejen entre los sujetos alrededor del espacio y de las prácticas espaciales, a distintas escalas y en distintas temporalidades, nos lleva a comprender mejor los resortes societales y políticos de las sociedades concernidas.

La exploración de esta cuestión me llevó a probar dos hipótesis principales. La primera consiste en plantear que los contextos de movilidad favorecen las "innovaciones territoriales", aunque sea sólo para responder a la novedad objetiva de las situaciones con las que se encuentran las personas, las familias y los colectivos durante sus desplazamientos. La segunda hipótesis plantea que estas innovaciones territoriales son una vía privilegiada de expresión pública para los que no disponen de capital suficiente para hacerse oír a través de los medios políticos institucionalizados: los que no tienen acceso a un capital económico negociable ; o los que no disponen de suficiente capital discursivo para garantizarles un mínimo de capacidad de negociación en las escenas públicas. Es decir, sugiero que el espacio funciona como un lenguaje político accesible a los que suelen estar excluidos de las arenas políticas instituidas. Esto no significa que las prácticas espaciales deban entenderse como un lenguaje específico de los subalternos. Recordemos, en efecto, que el control y el manejo del espacio siempre han sido instrumentos privilegiados de los poderes existentes. Gobernar, es primero conocer y controlar los recursos, entre ellos el espacio ; es implementar técnicas de poder basadas en el espacio y el territorio, como lo han señalado autores clásicos desde Raffestin 
(1980), Lacoste (1976), etc. (ver más adelante). Me inscribo en esta línea de interpretación con una matiz, pues no enfoco mi análisis hacia las prácticas espaciales de los actores dominantes sino hacia las de personas y colectivos que están en posición de subordinación, a veces incluso de franca dominación. Al igual que otros lenguajes, las prácticas espaciales se insertan en juegos de poder en los que, según los casos, pueden - o no - imponerse frente a prácticas y argumentos de los demás. Reconocer que el espacio funciona como lenguaje político no es, en sí, una novedad. En cambio, reconocer el potencial político de este lenguaje entre actores y sujetos cuya legitimidad territorial es sistemáticamente cuestionada, como en el caso de los migrantes, constituye una contribución que merece desarrollarse. Empezaré exponiendo los fundamentos teóricos de mi argumento, para luego analizar distintas configuraciones empíricas que permiten apoyar mis hipótesis.

\section{Recortar y categorizar el espacio, las tecnologías territoriales}

Desde los años 1970 existe un relativo consenso alrededor de nociones centrales en geografía: el espacio no es una categoría objetiva sino que éste sólo existe en cuanto está vivido, percibido, representado por individuos y colectivos sociales. Se transforma y reinterpreta cotidianamente por las poblaciones que lo explotan, lo viven, lo atraviesan (Frémont 1976). Reconocerlo llevó a plantear que el espacio se construye socialmente ; es un producto social (Lefevbre 1974). Hablar de espacio «construido» implica hablar de los actores de esta construcción, los agentes, los procesos de construcción (técnicas, tecnologías) y la historia, los tiempos.

Es así como una corriente importante de la geografía se dedicó a explorar las funciones políticas de la disciplina, en la medida en que ésta proporciona técnicas de gestión y manejo del espacio que son intrumentos de control y dominación. Después del famoso libro de Lacoste en 1976 («La geografía sirve, ante todo, a hacer la guerra »), otros autores han descrito como el control del espacio ha sido históricamente una prerrogativa del Estado que la ha ejercido a través la creación de institutos militares de geografía, la formación de personal técnico especializado, la monopolización de las cartografías nacionales y ciertas políticas públicas que han llevado a desigualdades e injusticias socio-espaciales (Reynaud,1981).

Otro paso importante consistió en reconocer que esta dimensión política no es solo instrumental, sino que es consustancial al espacio. El espacio es, en naturaleza, político (Lévy 1994). Si espacio y sociedad no existen separadamente, significa que, lógicamente, el espacio no solamente es socialmente construido sino que también participa en la construcción social. El espacio es producto social, pero también productor de sentido social.

Ahora bien, ¿de dónde viene esta calidad consustancialmente política del espacio, en la práctica? Viene de que vivir en el espacio es una experiencia humana universal ; implica la coexistencia y la obligación de convivir, es decir de "ver" y finalmente de comunicar y negociar con el otro, aunque solo sea para evitarle o eliminarle. En tanto que siempre una posición en el espacio siempre debe "negociarse", nunca es "natural". El espacio es el ámbito de experiencia de la pluralidad, de la copresencia necesariamente negociada ; este aspecto ha sido particularmente desarrollado en la antropología urbana que ve en la ciudad, aunque sólo sea por la densidad de población que la caracteriza, el ámbito privilegiado de la negociación espacial (Joseph 1998).

En las décadas de 1990 y 2000, se vuelve a enfatizar en la dimensión política del espacio, pero « lo político » es ahora visto desde otro ángulo, de una manera que rebasa la geopolítica de los años 1970 e insiste en la dimensión filosófica de la relación espacio-política. El espacio es doblemente político, en tanto que hace posible y visible el debate público, y en tanto que obliga a uno (individuo) a actuar a la vista del otro (Dikeç 2012). Es decir, el espacio (las prácticas en el espacio, del espacio) es condición y expresión de democracia. 
Desde la sociología política, se reconoce que el espacio es político en cuanto que es un "sistema de relaciones". Significa, prácticamente, que la espacialización hace posible ciertas puestas en relación, algunas sí y otras no, algunas más que otras. La relación (entre dos personas, colectivos, fenómenos) nunca es evidente, natural ni sistemática. Se construye en el proceso de subjetivación. La noción de subjetivación define el proceso de construcción de la relación sujeto / espacio, en la medida en que no existe sujeto fuera de un posicionamiento espacial, ni espacio sin sujeto social que lo conozca y lo identifique. La subjetivación no se construye desde el exterior ; se construye desde la acción y el posicionamiento ; la subjetivación es fundamento de la ciudadanía y de cualquier relación política (definida como relación entre sujetos legítimos) (Agier, 2013). Y siempre es espacializada.

Visto así, el manejo político del espacio ni es sistemáticamente una prerrogativa del poder y la autoridad como se había documentado ampliamente en los años 1980-90, ni tampoco es un recurso siempre movilizable por los subalternos en su movilización para la emancipación o la resistencia (Nicholls et al. 2011, Scott 2008). El espacio es más bien un campo de disputa, un lugar de posible imposición y también de posible protesta contra el orden establecido. Para Dikeç (2005) que retoma al filósofo Jacques Rancière, la naturaleza política del espacio viene de su capacidad de introducir polémica proponiendo nuevos principios (en su caso, igualdad y libertad). El espacio vale por su capacidad a (de) aportar sentido a las acciones, individuales y colectivas, sin utilizar volabulario explícito previo y fijo. Hay códigos pero gran latitud para interpretarlos.

Las prácticas redibujan tanto los sujetos como los espacios. Ambos adquieren calidades distintas en función de su interrelación : los pobladores de tal o cual porción de espacio se transforman en «tributarios» o «ciudadanos» en cuanto ésta se vuelve jurisdicción (municipio, estado, nación). Al revés, una lucha política o económica entre facciones facilmente adquiere expresiones espaciales que pueden desembocar en nuevas divisiones territoriales, el caso más evidente sigue siendo el de las luchas anticoloniales y de la creación de territorios políticos independientes. Es ahí donde la diferencia entre espacio y territorio se hace más pregnante. A diferencia del espacio, que es un campo de disputa a la vez material e imaterial (político, cultural, etc.), como lo acabamos de precisar, el territorio es una porción delimitada de espacio, sujeta a un proceso de apropiación por sujetos sociales y actores políticos, sean individuos (territorio corporal, territorio familiar) o, lo más comunmente, colectivos : los territorios de un pueblo, una empresa, un grupo étnico, una nación.

Históricamente, en la tradición política occidental, el modelo del estado-nación moderno westfaliano que se impone en el siglo XVII ha legitimado un orden territorial que se construyó sobre la adecuación entre espacio y población, territorio y pueblo, es decir una supuesta correspondencia entre una nación, un pueblo, un territorio nacional e, idealmente, una lengua común. Desde entonces, en este modelo occidental, gobernar consistió en gestionar estas dos entradas: el control del territorio (soberanía territorial, fronteras) y el control de la población (fiscalidad, registro civil). Para ello, la administración organizó e instituyó categorías, tanto de espacio como de población: jurisdicciones territoriales (municipios, distritos, departamentos o provincias, etc.); categorías demográficas (por edad, sexo, empleo, residencia, religión, etc.). En el acto de gobernar se toman en cuenta las interacciones entre las categorías de un orden (espacial) y las categorías del otro (poblacional) y las maneras en que evolucionan (por ejemplo: qué políticas públicas adoptar cuando se detecta la presencia de más pobres en tal lugar, de menos jóvenes en tal otro, etc.). Se podría pensar en la democracia como una permanente y necesaria reorganización entre distintos registros de clasificación: espaciales, identitarios, económicos, etc., siendo, cada una, una puesta en orden parcial del mundo que sólo cobra sentido respecto a los demás. Al contrario, 
el totalitarismo aparecería cuando la adecuación es total y fija entre espacio y población: cuando cada quien "está en su lugar" y nadie se mueve sin una buena razón y sin autorización.

Los contextos de movilidad son lugares de observación privilegiada de las interacciones entre espacio, población y el actuar político. La migración (comprendida en el sentido amplio de circulación y movilidades múltiples) significa ruptura en el espacio y también en el tiempo, o más bien en las temporalidades: afecta al tiempo cotidiano (tiempo del espacio doméstico que se transforma con la migración), al tiempo colectivo (ligado al espacio del pueblo o la comunidad que se deja o a la que se llega), al tiempo también de las pertenencias sociales ampliadas (los ritmos de la ciudadanía con las elecciones y las manifestaciones cívicas de un país o de otro, por ejemplo). En contexto de migración se rearticulan las escalas espaciales y temporales -así como sus relaciones- para dar lugar a nuevos significados de espacios y lugares que cuentan con una morfología renovada. Estas reorganizaciones no se hacen de manera natural, flexible y armoniosa. Se elaboran en medio de incertidumbres, desconocimientos, limitaciones, imposiciones $\mathrm{y}$, a menudo, violencia. En efecto las reorganizaciones espaciales movilizan jerarquías e introducen nuevos mecanismos de poder, cuestionan el orden establecido y llevan a reinventar mecanismos de hegemonía local, regional o nacional. Moldean nuevos sujetos sociales y políticos, y fronteras entre estos sujetos.

Estas propuestas teóricas tienen repercusiones metodológicas y empíricas muy concretas. Para entender las transformaciones en y del espacio hay que observar las prácticas espaciales, las tecnologías territoriales, las escalas de tiempos y espacios, los actores y sus comportamientos, las representaciones que se tiene de los lugares, territorios y espacios. También hay que entender el espacio en su materialidad y poder describir su ubicación, extensión, sustancia (lugar, área, zona, región, el vocabulario emic, etc.). Para lograrlo, se necesita combinar los enfoques de la geografía con una etnografía que contemple tanto los procesos estructurales como la agencia de los sujetos espacializados. Así se busca detectar y describir dispositivos territoriales originales con el fin de comprender mejor las lógicas de los actores y las escalas de acción que los respaldan. En las últimas décadas este enfoque se ha desarrollado principalmente en contextos urbanos marcados por una intensa movilidad y un fuerte impacto de los fenómenos de globalización. Así, los estudios sobre la "ciudad global" resultante de las circulaciones distinguen algunas formas ideales-típicas como la ciudad diaspórica, la ciudad fronteriza o la ciudad cluster (Besserer 2015), mientras que los estudios sobre las movilidades transnacionales pusieron en evidencia la existencia de enclaves étnicos comerciales y “territorios circulatorios" (Tarrius 2009; Parella, Valenzuela y Güell, 2015), entre otras posibles formas territoriales.

En este trabajo, propongo examinar algunas "configuraciones territoriales de movilidad" en las que es claramente el proceso de movilidad el que explica y condiciona la aparición de nuevas categorías territoriales. Presento sucesivamente tres casos: los territorios étnicos; los territorios ad hoc nacidos de coyunturas específicas ; las configuraciones territoriales en diáspora.

\section{Los territorios étnicos.}

Me refiero aquí a espacios tradicionalmente asociados a pueblos o comunidades pensadas como estables - las comunidades indígenas rurales- y que, en contextos de movilidad, se reconfiguran in situ, en los lugares de origen de los migrantes. Tomaré dos ejemplos, el del pueblo misak en Colombia y el del territorio comunal maya en Belice. Ambos casos son 
representativos de las dinámicas políticas y étnicas que, desde los años setenta del siglo pasado en América Latina, llevan a ciertos colectivos étnicos a reivindicar derechos específicos, en particular derechos territoriales asociados a los pueblos autóctonos. Sin embargo, aquí no me intereso tanto por las reclamaciones territoriales basados en la ancestralidad de una comunidad en un territorio dado, sino por la manera en que la noción de "territorios étnicos" se adapta a procesos recientes de movilidad y migración fuera del territorio "ancestral".

En Colombia, el pueblo misak (antes conocido como guambianos, unas 21.000 personas en 2005, que viven en la cordillera central en el departamento del Cauca) reivindica la autonomía de gestión de sus territorios ancestrales definidos, en otros argumentos, sobre la base de las tierras de resguardo asignadas por los gobiernos coloniales y posteriormente republicanos (siglo XIX). Para construir una legitimidad territorial portadora de autonomía política y cultural, las organizaciones étnicas misak movilizan la historia, la memoria, la autoctonía y la ancestralidad. La autonomía territorial se justificaría, en particular, por una construcción cultural muy específica del espacio que se ilustra en la figura 1. Según la visión misak, el territorio se expande "mediante 4 direccionales lingüísticas hasta llegar a expresar la redondez infinita (...) Con respecto a las nociones de espacio, la lengua guambiana siempre refiere al individuo a un punto o lugar de referencia, a partir del cual desarrolla su espacio hasta abarcar el territorio y el mundo, a este ejercicio los Misak lo llaman "desenrollar". Este punto o lugar de referencia es la casa, o el sitio de habitación del grupo de parentesco" (Duarte 2015: 289, tomado de Muelas 1993: 21).

Figura 1. La visión misak de la expansión del espacio" (Duarte 2015: 289, tomado de Muelas 1993: 21).

Esta lectura culturalista de las relaciones entre la comunidad étnica y su espacio de pertenencia es privilegiada por las organizaciones étnicas para justificar sus peticiones. También responde a las exigencias de las instancias de decisión gubernamental que piden a los grupos étnicos ofrecer pruebas de "especificidad cultural" para poder ser sujetos de políticas de reconocimiento de derechos con base en su diferencia cultural o étnica. Sin embargo, a finales del siglo XX, esta lectura queda sujeta a una fuerte presión debido a cambios estructurales que afectan las relaciones de las comunidades con sus espacios de vida. En efecto, la presión demográfica y los grandes cambios económicos (principalmente el declive de la actividad agrícola) suscitan la migración de personas misak fuera de sus territorios de origen, dirigiéndose ellas hacia las ciudades o hacia otras regiones del país. En este contexto, las autoridades tradicionales misak ampliaron sus concepciones de "espacios de vida". Se concibe ahora el pueblo misak como un "pueblo disperso", cuya territorialidad no implica continuidad espacial sino que integra potencialmente cualquier nueva localidad en la que residen los misak. El territorio misak se extendería a cualquier lugar habitado por un misak. Al mismo tiempo, aparece entre los dirigentes la necesidad de organizarse politicamente para adaptarse a esta nueva situación, constituyéndose en una confederación. La Confederación de territorios indígenas misak "representaría el paso necesario hacia la constitución de una Nación indígena Misak o Nuna Chak, en el interior de la nación 
colombiana" (Duarte 2015: 304). En este caso, es realmente la movilidad -aquí la migraciónla que, mediante nuevas prácticas espaciales, lleva al pueblo misak a inventar nuevas representaciones de sí mismo en el espacio y, por lo tanto, de su lugar en espacios más globales, en este caso espacios nacionales. Las prácticas espaciales se insertan en temporalidades múltiples que articulan la ancestralidad de la presencia (resguardos indígenas) con la actualidad de la migración laboral y combinan las legitimidades procedentes de ambos posicionamientos. La práctica espacial (aquí la dispersión) es la que crea un nuevo sujeto colectivo (la Confederación misak).

Encontramos un caso similar en Belice, donde los mayas del sur del país -principalmente hablantes de mopan y q'eqchi'- se organizan desde hace veinte años para luchar contra su marginalización, contra las concesiones que el gobierno otorgó a empresas forestales y petroleras sobre lo que consideran sus tierras y, en general, por el reconocimiento de sus derechos territoriales en una amplia región del distrito de Toledo. Reunidos en varias organizaciones (Toledo Alcaldes Association, Maya Leaders Alliance, Toledo Maya Cultural Council), los dirigentes mayas empezaron luchando por la protección de las tierras de pueblos que correspondían a las "indian reservations", las reservas coloniales que el gobierno colonial británico les habían asignado a principios del siglo XX. En este esquema de movilización, a cada localidad (village) le corresponde una tierra propia y una autoridad política reconocida (alcalde). Más recientemente, a raíz de su inserción en las redes internacionales de solidaridad con los pueblos autóctonos en América Latina, los dirigentes mayas actualizaron sus discursos y reclamos sobre los territorios. Las organizaciones elaboraron el concepto de "Maya homeland", el territorio maya ancestral o "tierras comunales" (communal land) asociadas al grupo maya en su conjunto (mopan y q'eqchi'). En esta concepción, el maya homeland no es la suma de las reservas territoriales otorgadas por el poder colonial sino que es un territorio unificado y contiguo que cubre el conjunto de lugares apropiados por gente maya. Se trata de una innovación discursiva y conceptual de primera importancia ya que abandona la noción de las "tierras de reservas" o de pueblos / localidades / villages, que son unidades separadas las unas de las otras, para promover la defensa de un territorio colectivo sin límites fijos que se adapta a las movilidades y los anclajes de los habitantes maya. Lo ilustra la cartografía del Maya Atlas, una magnífica obra realizada por las comunidades mayas de Toledo en su lucha por el territorio maya, publicada en 1997. Una de las láminas del atlas representa las tierras de pueblos (land villages) como tantos territorios distintos unos de otros. Otra lámina del mismo Atlas ilustra la misma cuestión de las tierras pero con otro código gráfico : dibuja un área unificado que viene a englobar y sobreponerse a las anteriores tierras de pueblos, bajo otra apelación: es el maya homeland o communal lands.

La adopción de una nueva categoría de espacio - el maya homeland - da cuenta de la evolución política, demográfica y geográfica del asentamiento maya en el sur de Belice. La noción es construida por y para un nuevo actor colectivo -el pueblo maya- que no preexistía a estas innovaciones. Por supuesto, ya existían campesinos y pueblos mayas, lenguas mayas (mopan y q'eqchi'), organizaciones maya, pero no un "pueblo maya" capaz de tomar la palabra y exigir derechos específicos frente al Gobierno nacional y a las organizaciones internacionales (en particular, la Corte Interamericana de los Derechos Humanos, CIDH). Ahora es el caso. Aquí no es la práctica espacial (migración) en sí misma la que modifica los contornos del sujeto social colectivo y sus espacios, sino las elaboraciones discursivas y cartográficas cuyo efecto performativo es muy eficaz (Wainwright y Bryan 2009). 
Una segunda configuración territorial de movilidad corresponde a casos de construcción territorial explícitamente pensada para solucionar un problema preciso y concreto, por lo que los llamo territorios ad hoc. En Colombia, Jessica Corredor (2015) documentó este fenómeno en el caso de los desplazamientos forzados que se multiplicaron en el país en los años noventa. Después del trauma del desplazamiento, alguno.as campesino.as ambicionaron volver a vivir sobre sus tierras y se organizaron para lograrlo, a pesar de la presencia de actores externos que los acosan cotidianamente : los paramilitares que quieren expulsarlos para controlar el espacio, algunas plantaciones agroindustriales que pretenden explotar las tierras sin restricción o el propio gobierno que busca imponer sus propias instituciones y autoridad. Concretamente, en el departamento del Chocó, en la parte noroccidental del país, los desplazados lucharon para regresar a su espacio de origen, reinstalarse y crear las condiciones materiales y políticas de su protección. Para ello, una vez reinstalados en casas provisionales, empezaron a reorganizar la vida social colectiva y la protección de los habitantes. Cercaron las áreas de residencia y de trabajo, controlaron entradas y salidas, registraron usuarios con derechos, etc. Con el apoyo de las $\mathrm{ONG}$, exigirieron que el gobierno los recibiera para negociar garantías y políticas de acompañamiento en sus nuevas instalaciones. Así es como nacieron algunas áreas llamadas zonas humanitarias $(\mathrm{ZH})$ y zonas de biodiversidad (ZB), que son espacios concretos, delimitados, cuyo acceso se controla y se reserva estrictamente a los desplazados y sus "acompañantes", o sea las ONGs humanitarias. Las zonas humanitarias $(\mathrm{ZH})$ son las áreas de residencia, mientras las zonas de biodiversidad (ZB) corresponden a parcelas de actividad agrícola. Ambas son pensadas para ser espacios de refugio que se sustraen al conflicto y sus actores armados (paramilitares, ejército, narcotraficantes, guerrillas, entre otros). Al instituir estas zonas, tanto en el terreno como en el discurso, los desplazados se dotaron de una territorialidad original que les permite resistir a las presiones de los actores externos.

Las zonas humanitarias y de biodiversidad se conviertieron en soportes de la movilización política en pro del derecho al retorno y de la protección de la vida. Son territorialidades políticamente instrumentalizadas por actores que, a partir de algunos lugares y espacios delimitados, movilizan un amplio espectro de recursos a nivel local/regional y, sobre todo, nacional e internacional (las ONG humanitarias). Para los campesinos desplazados, la inscripción en un espacio local (las ZH y ZB) da acceso a una palabra ampliada que se hace escuchar mucho más allá del lugar de enunciación. Permite exigir -y obtener- de los actores situados afuera de las "zonas" el respeto de los que están ubicados adentro de las mismas. La legitimidad territorial adquirida mediante el control material de un espacio local, aunque fuera de tamaño muy reducido, -su delimitación, su control, su uso-, sirve para ganar poder de negociación frente a las autoridades en otros registros. A partir de las zonas humanitarias y las zonas de biodiversidad, los protagonistas locales del Chocó hasta pudieron pretender influir en políticas públicas relativas al trato de las víctimas del desplazamiento forzado en todo el país. La convergencia entre una demanda local territorializada y una lógica de negociación más global hizo posible la emergencia de una dinámica política nacional.

Este ejemplo ilustra la manera en que categorías de espacios instituidas (las "zonas") remiten a prácticas de intervención asociadas a colectivos sociales ("los desplazados retornados"), así como a métodos específicos de gobierno (las autoridades de los grupos de retornados y las ONG acompañantes). Es muestra de un registro político calificado por Planel de "derogatorio", en el sentido en que, en ciertos espacios delimitados, el gobierno instituye normas particulares de regulación (Planel 2015). Esta autora insiste en que la excepción territorial es, en sí, un dispositivo de control de la población que ahí reside. A esto sólo añadimos, con el caso de los territorios ad hoc, que este tipo de dispositivo territorial derogatorio no solo le sirve a los dominantes para imponer su propio orden, sino que puede 
promoverse también como recurso por parte de actores subalternos que encuentran ahí argumentos de resistencia (Hoffmann 2016, Nicholls et al. 2011). Encontramos otro ejemplo de expresión territorial de la resistencia en las movilizaciones sociales que, en Francia, se dan en torno a conflictos ambientales en áreas calificadas de "ZAD". El acrónimo ZAD es inicialmente una sigla empleada por las autoridades para señalar "Zonas de ordenamiento diferido" (zones d'aménagement différé), es decir, zonas cuyo uso se restringe ya que se "reservan" para operaciones de ordenamiento a futuro (aeropuerto, presa, etc.). Posteriormente este significado fue desviado por los militantes ecologistas y anticapitalistas que se movilizan en contra de dichos proyectos de ordenamiento territorial. Ellos ocupan las zonas reservadas y las renombran según el mismo acrónimo ZAD pero que significa ahora "Zonas por defender" (Zones à défendre). Ellos mismos se transforman entonces en "zadistes". La palabra entró en el vocabulario usado en los medios de comunicación y hasta figura en el diccionario (Petit Robert 2016). Es el espacio (ZAD) el que define los contornos del actor político (zadiste) ; es la zona que define la función a defender, y en consecuencia el colectivo político zadista.

\section{El caso de las configuraciones en diáspora}

Finalmente, un tercer caso de configuración territorial en movilidad, más conocido, es el de los espacios diaspóricos, en los que la movilidad va acompañada de un cambio radical de la territorialidad, consecuencia de la pérdida traumática de la relación con el territorio de origen. Numerosos trabajos han subrayado la intensidad de los traumatismos de los exilios forzosos (Hovanessian 2007 (2011), Chivallon 2004). Otros autores destacan la manera en que, en contextos de globalización, la circulación acelerada de personas y sujetos lleva a la constitución de diásporas culturales y de nuevos "paisajes" (los scapes de Appadurai, 1996). En efecto desde hace varias décadas, el concepto de diáspora se ha extendido a los casos de migraciones masivas; califica fenómenos que afectan a numerosas regiones del mundo y reorganizan las territorialidades, tanto en las zonas de salida como en las de llegada, y sus interacciones. Para América Latina, los trabajos sobre la migración hacia los Estados Unidos pusieron en evidencia lógicas de migración transnacional (Glick-Schiller, Bash y BlancSzanton 1995) que desembocan en la construcción de comunidades transnacionales (Portes, Guarnizo y Landolt, 2003) cuya existencia se formaliza a veces en el vocabulario popular (Nezayork, Oaxacalifornia).

Las relaciones entre migrantes y residentes suscitan procesos de recomposición de los espacios de vida y de los colectivos de pertenencia, siendo los migrantes tanto de aquí como de ahí, de aquí o de ahí, de aquí gracias a ahí, de aquí dentro de ahí, etc. Ya no se oponen los lugares de salida y los lugares de llegada sino que se transforman unos y otros gracias a nuevas articulaciones e interpenetraciones. La multiplicidad de las formulaciones remite a la multiplicidad de los fenómenos que sustentan estas innovaciones espaciales.

Unos remiten al campo cultural, como lo ilustra la realización de eventos culturales gemelos en los lugares de origen y de residencia, en los dos países : típicamente la fiesta patronal del pueblo mexicano que se adapta a la localidad de residencia de los emigrantes integrando imágenes y símbolos de los Estados Unidos, por ejemplo su bandera. Otro caso lo ilustra la adaptación de algunos rituales de curación que se siguen celebrando en el pueblo de origen, aun en ausencia de los pacientes que están en migración pero cuya "presencia" se garantiza a través de su teléfono móvil. 
Otros fenómenos dan cuenta de esta territorialidad compleja que ofrece nuevas oportunidades económicas. Así en Playa Vicente (Veracruz) a principios de los años 2000, una señora se dedicaba a la venta de "pan hecho en casa" a los migrantes residentes en Estados Unidos. Se beneficiaba de una visa que le permitía viajar muy frecuentemente, trayendo de vuelta noticias de los migrantes y ropa de segunda mano. El comercio transnacional de bienes íntimos de escaso valor mercantil pero muy valorados por los migrantes (objetos de la vida corriente o bienes personales, alimentos preparados en el pueblo) resultaba lo suficientemente redituable -junto con el comercio de ropa- para pagar los pasajes aéreos. En este esquema los bienes transitan de un lado a otro de la frontera, en los dos sentidos, y mantienen un potente vínculo entre residentes de los distintos lugares. Estas situaciones resultan en su mayoría de iniciativas personales o familiares, a menudo poco estabilizadas y poco institucionalizadas. No solo ponen en relación los lugares de origen y de residencia de los emigrantes, sino que crean nuevos espacios, materiales o inmateriales, y nuevas prácticas económicas o de sociabilidad (la relación con el especialista ritual, los nuevos espacios rituales cívicos o religiosos, los espacios físicos y económicos de circulación). Varios autores desarrollaron a este respecto el concepto de comunidades transnacionales que serían fruto de "procesos transnacionales subalternos" resultantes de una "globalización desde abajo" en oposición a la globalización impuesta desde arriba (Besserer 2015: 8). No obstante, conviene matizar este entusiasmo y recordar que estos procesos transnacionales no se inventan en contextos de armonía y consenso. Al contrario, como cualquier innovación, provocan rivalidad entre registros de autoridad y entre los sujetos y actores sociales que los experimentan: entre los que se quedan en el pueblo y los que emigraron, entre los que "lo han logrado" y tienen recurso y los que no lo tienen, entre los que adoptan las nuevas normas de vida (familiar, de pareja, de género, de trabajo, de religión) y los que no cambian, etc. Las nuevas prácticas se dan en lugares específicos que estructuran el espacio de manera distinta a la anterior: nuevas iglesias, nuevos comercios, nuevas casas, nuevas organizaciones políticas, etc. Aunque el fenómeno ya está bastante documentado en la literatura especializada, conviene recordar las conclusiones que se pueden extraer para sentar nuestra argumentación: las prácticas del espacio desarrolladas en contextos de diáspora visibilizan nuevos colectivos, como pueden ser "los emigrantes" que viven lejos de sus lugares de origen, "los ilegales" que comparten la experiencia traumatizante de la migración sin tener los papeles legales, "los paisanos" sujetos de las políticas públicas mexicanas, "los conversos" miembros de tal o cual nueva iglesia, etc. Las fronteras entre estas nuevas identidades son múltiples y no coinciden unas con otras. De la misma manera que no hay ruptura definitiva entre espacios y actores migrantes y no migrantes, tampoco hay adecuación sistemática entre espacios y actores sociales en diáspora. La configuración territorial en diáspora se caracteriza más bien por yuxtaposiciones, a veces intersecciones entre grupos de actores que comparten lugares para acciones o tiempos específicos (de residencia, de trabajo, de práctica religiosas..), sin constituir territorios estabilizados.

\section{Debate y conclusiones}

La primera hipótesis que había planteado en la introducción queda sobradamente consolidada, en realidad era bastante intuitiva. La movilidad crea nuevas territorialidades y a veces, incluso, nuevos territorios. Los ejemplos han mostrado la capacidad de los protagonistas para crear territorio a partir de competencias vinculadas a su movilidad, e implementar acción política usando herramientas geográficas. Ante una movilidad obligada, por ejemplo, los protagonistas inventan categorías geográficas que les sirven para expresar sus nuevos posicionamientos en el registro político. El espacio funge como recurso para construirse e 
identificarse. Hemos visto en el ejemplo de las zonas humanitarias y de biodiversidad cómo la privación violenta de espacio (las personas desplazadas) generó nuevas prácticas territoriales (el regreso en las zonas humanitarias) que a su vez instituyeron y legitimaron nuevos colectivos sociales y políticos. El espacio remite al grupo que lo crea, lo comprende, y a la vez se crea a él mismo (la nación misak, el colectivo de víctimas del desplazamiento forzado, los zadistas).

La relación del sujeto con el lugar/espacio es constitutiva de la relación de uno con sí mismo y sus vecinos. Cimenta las fronteras simbólicas y políticas que organizan el mundo en cada sociedad o grupo (Gupta y Ferguson 1997). Estas fronteras remiten generalmente a procesos de separación "nosotros/ellos" e incluso de discriminación "nosotros contra ellos". Históricamente, en los modelos occidentales, la frontera entre estados- naciones ha sido la más pregnante y pretendió ser a la vez social, económica, cultural y política. A finales del siglo 20, también se reconoció el significado político de otras territorialidades, específicamente en el caso de los grupos étnicos que reivindican subjetivaciones territorializadas en tanto Naciones originarias, Pueblos autóctonos, pueblos indígenas, pueblos maya de Guatemala, entre otros ejemplos. En ambos casos -las fronteras nacionales y las territorialidades étnicas- la gestión de los lugares y los territorios ocupa un papel estratégico en el aprendizaje político, cultural y social de los protagonistas de la historia. Transformar los espacios es transformar a los sujetos y a su relación unos con otros.

En la gobernanza por medio del espacio, no todo está controlado por un grupo o colectivo en particular. Y allí es donde nuestra segunda hipótesis ha mostrado su pertinencia y nos ha ayudado a interpretar las situaciones observadas. El espacio puede ser efectivamente un recurso accesible para los sin-voz, un medio de expresión para los que no tienen la práctica ni los medios de hacerse oír en las escenas públicas. El espacio ofrece la oportunidad de "decirse" sin tener que elaborar un discurso verbal normalizado. Es un lenguaje accesible para los que no dominan los códigos utilizados en las transacciones comunmente llevadas a cabo entre actores públicos.

Interesándose por la arquitectura, A.Lévy también veía ahí un lenguaje, sosteniendo que esta propuesta implicaba dos postulados: " (I) el espacio no necesita hablarse para significar, sino que significa directamente; (ii) el espacio significa algo más que él mismo, algo más que su materialidad física" (Lévy 2008). Si el espacio significa por sí mismo, sin necesidad de traducción, entonces es necesario atenderse a sus características propias, materiales (estructura, tamaño, composición, ubicación, etc.), para entender este significado. Es decir, el espacio no es sólo metafórico, sino que saca su eficacia discursiva de su materialidad oponible a la experiencia práctica de cada uno. Muy concretamente, la materialidad del espacio impone negociaciones en caso de copresencia, lo hemos visto en los ejemplos mencionados. $\mathrm{Y}$ al mismo tiempo, con o sin conflicto, en un mismo espacio concreto, las territorialidades suelen ser múltiples y competir unas con otras. Las territorialidades superpuestas activan regímenes de legitimidad y autoridad que entran en rivalidad los unos con los otros (Agnew y Oslender, 2011). En este sentido, el espacio significa también mucho más allá que su materialidad. No hay transcripción directa del espacio geográfico sobre lo político, no hay proyección inmediata de uno sobre otro (por ejemplo, en la relación entre control territorial y control político). Es decir, no estamos frente a un nuevo determinismo espacial. Al contrario, el espacio abre el abanico de opciones. Las prácticas espaciales componen un lenguaje político en cuanto que posibilitan rupturas con el orden social imperante, como lo propone Dikeç, incluso sin tener que afirmarlo verbalmente. El espacio es intrínsecamente político no solo como recurso negociable, sino como lenguaje potencialmente 
subversivo. Ahora bien, las prácticas espaciales no son, como tales, subversivas ; pero llevan en ellas este potencial, son el resultado de luchas simbólicas o materiales (Lussault 2009). Como lo mostró el ejemplo de los territorios ad hoc como las ZH/ZB o las ZAD, las prácticas espaciales se vuelven luchas que activan procesos hegemónicos de construcción de la autoridad y del poder, mismos que rebasan de mucho el ámbito geográfico de los espacios concretos de donde surgen.

Bibliografía

Agier, Michel. 2013. La Condition cosmopolite: l'anthropologie à l'épreuve du piège identitaire. Paris, La Découverte.

Agnew John and Oslender Ulrich, Overlapping territorialities, sovereignty in dispute: empirical lessons from Latin America, 2011, pp. 141-163 in Walter Nicholls, Byron Miller and Justin Beaumont (editors), Spaces of Contention: spatialities and social movements Aldershot: Ashgate

Appadurai, Arjun. 1996. Modernity at Large. Cultural Dimensions of Globalisation, Minneapolis, University of Minnesota Press.

Besserer, Federico, Presentación del número, Alteridades [en linea] 2015, 25 (JulioDiciembre) : [Fecha de consulta: 22 de noviembre de 2016] Disponible en: $<$ http://www.redalyc.org/articulo.oa? $\mathrm{id}=74743764001>$

Chivallon Christine. 2004. La diaspora noire des Amériques. Expériences et théories à partir de la Caraïbe. Paris, Éditions du CNRS (Collection Espaces et Milieux), 258 p.

Corredor Jessica. 2015. «Exercer sa citoyenneté par le droit à la terre: les Zones Humanitaires et de Biodiversité dans le Bajo Atrato en Colombie », thèse sociologieanthropologie, Université Paris Diderot, noviembre 2015

Dikeç, Mustafa, « Space, politics, and the political », Environment and Planning D: Society and Space. Apr 2005, 23, 2, p. 171-188

Dikeç, Mustafa, june 2012, «Space as a mode of political thinking », Geoforum. 43, 4, p. 669-676

Duarte, Carlos. 2015, «(Des)encuentros en lo público. Gobernabilidad y conflictos interétnicos en Colombia ». Thèse de doctorat en sociologie, Paris III, IHEAL.

Frémont, Armand. 1976. La Région, espace vécu, Paris, Flammarion.

Glick Schiller Nina, Basch Linda and Blanc-Szanton, Cristina. 1995. « From Immigrant to Transmigrant: Theorizing Transnational Migration », Anthropology Quarterly, 68 (1) (Jan.): 48-63.

Gupta, Akhil and Ferguson, James. 1997. « Beyond "culture" : space, identity, and the politics of difference ", p33-51 in Culture, Power, Place Explorations in Critical Anthropology, Gupta, Akhil and Ferguson, James (eds), Durkham and London, Duke University Press. 
Harvey, David. 1989. The condition of postmodernity, Oxford, Basil Blackwell.

Hoffmann, Odile. 2016. «Territoires et innovations politiques en contextes multiculturels (Colombie, XX et XXème siècles) », p55-74 in France Guérin-Pace et Evelyne Mesclier (coord.) Territoires et mobilisations contemporaines. Regards sur un phénomène planétaire. Paris, Karthala-CIST.

Hovanessian Martine "Diasporas et identités collectives », Revue Hommes et Migrations. $\mathrm{N}^{\circ} 1265$, janvier-février 2007, Mis à jour le : 08/06/2011, http://www.hommes-etmigrations.fr/index.php?/numeros/diaspora-armenienne/2153-diasporas-et-identitescollectives

Joseph, Isaac. 1998. La ville sans qualités, Éditions de l'Aube.

Lacoste, Yves. 1976. La Géographie ça sert d'abord à faire la guerre, Paris, La Découverte.

Lefebvre, Henri. 1974. La production de l'espace, Paris: Anthropos.

Lévy, Albert «Sémiotique de l'architecture», Actes Sémiotiques [En ligne]. 2008, n 111. Disponible sur : < http://epublications.unilim.fr/revues/as/2993> (consulté le 03/05/2016)

Lévy, Jacques. 1974. L'espace légitime. Sur la dimension géographique de la fonction politique. Paris, Fondation des Sciences Politiques, 1994.

Lussault, Michel. 2009. De la lutte des classes à la lutte des places, Paris, Grasset.

Melé Patrice. 2008., "Conflits et controverses : de nouvelles scènes de production territoriale ? \ in Isabelle Garat, Raymonde Séchet, Djémila Zeneidi (dir.), Espaces en (trans)action, Rennes, PUR, p. 239-250.

Muelas Hurtado, Bárbara. 1993. "Relación tiempo-espacio en el pensamiento guambiano". Tesis de Maestría en Lingüística y Español, Universidad del Valle, Cali Colombia.

Nicholls, Walter, Miller Byron, Beaumont Justin. 2011. «Conceptualizing the spatialities of social movements », p. 6-26 in Walter Nicholls, Byron Miller and Justin Beaumont (editors), Spaces of Contention: spatialities and social movements Aldershot: Ashgate

Parella, Sonia, Valenzuela García, Hugo, Güell, Berta. 2015. «La economía étnica en perspectiva: del anclaje a la fluidez en la urbe global », Alteridades [en linea] 2015, 25 (JulioDiciembre) : [Fecha de consulta: 22 de noviembre de 2016] Disponible en: $<$ http://www.redalyc.org/articulo.oa?id=74743764004> ISSN 0188-7017

Planel Sabine. 2015. "Espaces autoritaires, espaces (in)justes?” Justice Spatiale / Spatial Justice, $\mathrm{n}^{\circ} 8$ juillet 2015

Portes Alejandro, Luis Guarnizo y Patricia Landolt (coord.), 2003. La Globalización desde abajo: transnacionalismo inmigrante y desarrollo : la experiencia de Estados Unidos y América latina. México. Miguel Angel Porrúa-FLACSO

Raffestin, Claude. 1980. Pour une géographie du pouvoir. Paris, Ed. LITEC. 
Reynaud, Alain. 1981. Société, espace et justice. Inégalités régionales et jsutice socio-spatiale, Paris, PUF.

Scott, James. 2008. La domination et les arts de la résistance. Fragments du discours subalterne, Paris, ed. Amsterdam.

Swyngedouw E., 2011, "Interrogating post-democratization: Reclaiming egalitarian political spaces." Political Geography 30, no. 7(2011) : 370-380

Tarrius Alain. 2009. "Intérêt et faisabilité de l'approche des territoires des circulations transnationales », chapitre 3 en "Les circulations transnationales. Lire les turbulences migratoires contemporaines ", sous la direction de Geneviève Cortes et Laurent Faret, Paris, Armand Colin.

TMCC, Toledo Maya Cultural Council and Toledo Alcaldes Association. 1997. Maya Atlas. The struggle to preserve maya land in southern Belize. Belize.

Wainwright, Joel, and Joe Bryan. "Cartography, Territory, Property: Postcolonial Reflections on Indigenous Counter-Mapping in Nicaragua and Belize." Cultural Geographies 16 (2009): 153-78. 